\title{
Occult lymph node metastases in patients without residual muscle-invasive bladder cancer at radical cystectomy with or without neoadjuvant chemotherapy: a nationwide study of 5417 patients
}

\author{
L. M. C. van Hoogstraten ${ }^{1,4}$ (D) E. J. van Gennep ${ }^{2}$. L. A. L. M. Kiemeney ${ }^{3,4} \cdot$ J. A. Witjes ${ }^{3}$. C. S. Voskuilen ${ }^{5} \cdot$ M. Deelen $^{6}$. \\ L. S. Mertens ${ }^{5} \cdot$ R. P. Meijer ${ }^{7}$ J. L. Boormans ${ }^{8}$. D. G. J. Robbrecht ${ }^{9} \cdot$ L. V. Beerepoot ${ }^{10} \cdot$ R. H. A. Verhoeven ${ }^{1,11}$. $^{3}$ \\ T. M. Ripping ${ }^{1}$ - BlaZIB Study Group • B. W. G. van Rhijn ${ }^{12}$ - K. K. H. Aben ${ }^{1,4}$. T. J. N. Hermans ${ }^{13}$
}

Received: 21 July 2021 / Accepted: 9 September 2021 / Published online: 28 September 2021

(C) The Author(s) 2021

\begin{abstract}
Purpose Little is known about the prevalence of occult lymph node metastases (LNM) in muscle-invasive bladder cancer (MIBC) patients with pathological downstaging of the primary tumor. We aimed to estimate the prevalence of occult LNM in patients without residual MIBC at radical cystectomy (RC) with or without neoadjuvant chemotherapy (NAC) or neoadjuvant radiotherapy (NAR), and to assess overall survival (OS).

Methods Patients with cT2-T4aN0M0 urothelial MIBC who underwent RC plus pelvic lymph node dissection (PLND) with curative intent between January 1995-December 2013 (retrospective Netherlands Cancer Registry (NCR) cohort) and November 2017-October 2019 (prospective NCR-BlaZIB cohort (acronym in Dutch: BlaaskankerZorg In $\underline{B}$ eeld; in English: Insight into bladder cancer care)) were identified from the nationwide NCR. The prevalence of occult LNM was calculated and OS of patients with $<(y)$ pT2N0 vs. $<(y)$ pT2N+ disease was estimated by the Kaplan-Meier method.

Results In total, 4657 patients from the NCR cohort and 760 patients from the NCR-BlaZIB cohort were included. Of 1374 patients downstaged to $<(y)$ pT2, 4.3\% $(N=59)$ had occult LNM $4.1 \%(N=49)$ of patients with cT2-disease and 5.6\% $(N=10)$ with cT3-4a-disease. This was $4.0 \%(N=44)$ in patients without NAC or NAR, 4.5\% $(N=10)$ in patients with NAC, and $13.5 \%(N=5)$ in patients with NAR but number of patients treated with NAR and downstaged disease was small. The prevalence of $<(y)$ pT2N+ disease was $4.2 \%(N=48)$ in the NCR cohort and 4.6\% $(N=11)$ in the NCR-BlaZIB cohort. For patients with $<(y)$ pT2N+and $<(y)$ pT2N0, median OS was 3.5 years (95\% CI 2.5-8.9) versus 12.9 years (95\% CI 11.7-14.0), respectively.

Conclusion Occult LNM were found in $4.3 \%$ of patients with cT2-4aNOM0 MIBC with (near-) complete downstaging of the primary tumor following RC plus PLND. This was regardless of NAC or clinical T-stage. Patients with occult LNM showed considerable worse survival. These results can help in counseling patients for bladder-sparing treatments.
\end{abstract}

Keywords Bladder cancer $\cdot$ Neoadjuvant chemotherapy $\cdot$ Downstaging $\cdot$ Lymph node metastases $\cdot$ Radical cystectomy

The members of the BlaZIB study group are given in acknowledgements section.

L. M. C. van Hoogstraten and E. J. van Gennep are the co-first authors.

\section{K. K. H. Aben and T. J. N. Hermans shared senior authorship.}

L. M. C. van Hoogstraten

l.vanhoogstraten@iknl.nl

Extended author information available on the last page of the article

\section{Introduction}

The standard treatment for clinically node-negative muscleinvasive bladder cancer (MIBC) is radical cystectomy (RC) and pelvic lymph node dissection (PLND) with cisplatinbased neoadjuvant chemotherapy (NAC) in fit patients [1]. An alternative for RC is trimodality therapy (TMT) [1]. Transurethral resection (TUR) with or without external beam radiation therapy (EBRT) is considered inferior to RC or TMT [1,2], whereas TUR with or without systemic 
chemotherapy has the potential to be curative in selected cases [3-5]. The prevalence of occult lymph node metastases (LNM) at RC plus PLND is approximately $25 \%$ and as such, PLND is associated with improved survival in these patients $[6,7]$. In contrast, PLND or treatment of the lymph nodes is not part of the TMT protocol [2].

A recent Dutch population-based study including 4508 patients with cT2NOM0 urothelial MIBC showed that downstaging to non-MIBC was present in $25 \%$ after upfront RC and in 43 and $33 \%$ after NAC and neoadjuvant radiation (NAR), respectively [8]. In general, it is still not possible to accurately predict downstaging by TUR. Therefore, RC with PLND remains the standard of care. In selected cases or due to patient refusal, one might not always proceed to RC, CMR or EBRT [3, 4]. A clinical complete response after TUR-only or TUR combined with systemic chemotherapy cannot reliably be concluded based on a combination of Re-TUR, negative cytology and cross-sectional imaging. However, these diagnostics are often performed in daily practice in attempting to confirm a so called "pT0-status" in patients who prefer bladder preservation $[3,4,9,10]$. In these patients, PLND for the assessment of nodal invasion is not routinely performed and the prevalence of occult metastatic disease and the potential role of PLND in this particular group has not been clearly demonstrated [11].

In a recent retrospective cohort of patients treated with NAC plus RC, 4.9 and $5.4 \%$ of patients with ypT0 and ypTa/ is/1 disease had occult LNM [11]. This was irrespective of NAC or initial clinical T-stage. To our knowledge, other studies on this subject are not available. Therefore, the aim of this population-based study is to estimate the prevalence of occult LNM in patients without residual MIBC at RC, stratified by treatment with or without NAC and to assess OS in patients with and without occult LNM.

\section{Materials and methods}

\section{Patients}

Patients diagnosed with cT2-4aN0M0 urothelial bladder carcinoma (BC) who underwent RC plus PLND with or without NAC or neoadjuvant radiotherapy (NAR), between January 1st 1995 and December 31st 2013 (retrospective NCR cohort, data already available from Hermans et al. [8]) and between November 1st 2017 and October 31st 2019 (prospective NCR-BlaZIB cohort) were selected from the Netherlands Cancer Registry (NCR). The NCR-BlaZIB cohort consisted of patients included in the ongoing Dutch nationwide population-based prospective BlaZIB study (BlaaskankerZorg In Beeld, translation: Insight into Bladder Cancer Care) [12], which is embedded in the NCR. Patients who underwent a partial cystectomy or salvage cystectomy, or in whom PLND was not performed were excluded. Patients with histology other than UC as the main component were also excluded (Supplementary Fig. 1).

\section{The Netherlands Cancer Registry}

The NCR is a nationwide population-based registry collecting data on all newly diagnosed malignancies in the Netherlands. Identification is mainly based on notification from the nationwide network and registry of histopathology and cytopathology in the Netherlands (PALGA) [13]. Well-trained data managers of the NCR collect clinical data on predefined patient, tumor, and treatment characteristics from the individual patient files at each hospital. In the NCR, topography and morphology are classified according to the International Classification of Diseases for Oncology (ICD-O) [14]. Tumor stage is classified according to the TNM system [15]. Clinical staging was based on physical examination, findings at cystoscopy and TUR, computed tomography (CT-) scan of the abdomen/ pelvis and chest imaging (at least a chest X-ray).

In a previous study, all pathology reports of patients from the NCR cohort 1995-2013 were reviewed (TH, MD, CV, LM) after linkage with PALGA since pathological downstaging at $\mathrm{RC}$ to non-MIBC was not registered in the NCR as a standard item before 2017 [8]. For the NCRBlaZIB cohort, information on pathological downstaging was prospectively collected. Changes in TNM classifications over time (e.g., changes within pT2-stage) were irrelevant for our study outcomes [15]. Due to changes in the classification for nodal disease, it was only possible to categorize patients into node-negative (pN0) and nodepositive disease $(\mathrm{pN}+)$.

\section{Statistical analyses}

The numbers and percentages of occult LNM in patients without and with $(y)$ NAC and complete [(y)pT0] or partial downstaged [(y)pTa/is/1] primary tumors were calculated. The Kaplan-Meier method was applied to calculate median overall survival (OS) in patients with $(y)$ pT0N0 vs. (y)pT0N+disease and $<(y)$ pT2N0 vs. $<(y)$ pT2N+disease. Due to the limited number of patients, it was not possible to further stratify results by NAC or NAR. Date of RC was taken as start of follow-up. End of follow-up was defined as last date of follow-up or death, whatever came first. Logrank tests were used to compare survival distributions. Statistical analyses were performed with SAS version 9.4 (SAS Institute, Cary, North Carolina, USA). $P$-values $<0.05$ were considered statistically significant. 


\section{Results}

In total 5417 patients with cT2-4aN0M0 urothelial MIBC who underwent RC and PLND were analyzed. From the retrospective NCR cohort, 4657 patients were included and from the prospective NCR-BlaZIB cohort, 760 patients were included (Supplementary Fig. 1). Compared to the NCR cohort, patients in the NCR-BlaZIB cohort were older ( 70 versus 67 years) and more often had locally advanced disease (cT3/4a in $29.7 \%$ versus $18.2 \%$ ) (Supplementary Table 1$)$. In the earlier NCR cohort, NAC and NAR were applied in $6.4 \%(N=298)$ and $2.2 \%(N=104)$ of patients, respectively. This was $28.3 \%(N=215)$ and $0.5 \%(N=4)$ in the NCR-BlaZIB cohort.

In $18.7 \%(N=1013)$ of all PLND specimens LNM were found. In 1,374 patients downstaged to $<(y)$ pT2, $4.3 \%$ $(N=59)$ had occult LNM. In patients downstaged to $(y)$ pT0 or $(y) \mathrm{pTa} / \mathrm{is} / 1, \mathrm{LNM}$ were present in $4.1 \%(N=33)$ and $4.6 \%(N=26)$, respectively (Table 1$)$. In patients with cT2 and cT3-4a disease downstaged to $<(y)$ pT2, LNM were present in $4.1 \%(N=49)$ and $5.6 \%(N=10)$ $(p=0.3705)$, respectively. Stratification by NAC (upfront $\mathrm{RC}$ vs. NAC $+\mathrm{RC}$ ) resulted in comparable percentages of $<$ ypT $2 \mathrm{~N}+$ and $<\mathrm{pT} 2 \mathrm{~N}+$ disease in $4.5 \%(N=10)$ and $4.0 \%(N=44)$ of patients $(p=0.7093)$. In 108 patients who received NAR, 5 out of $37(13.5 \%)$ had LNM with <ypT2 at RC. The prevalence of $<(y)$ pT2N+disease was similar over time, $4.2 \%(N=48)$ in the NCR cohort and $4.6 \%$ $(N=11)$ in the NCR-BlaZIB cohort.

Patients with LNM following complete downstaging of the primary tumor $[(y) \mathrm{pTON}+]$ showed inferior OS versus patients with complete downstaging without LNM [(y)pT0N0] $(p<0.001)$. Median OS was $3.4(95 \%$ CI $1.7-7.0)$ vs. 14.1 years $(95 \%$ CI $12.9-17.1)$ (Fig. 1a). This association was also seen in patients with downstaging to non-MIBC $[<(y)$ pT2] $(p<0.001)$. The median OS was 3.5 (95\% CI 2.5-8.9) vs. 12.9 (95\% CI 11.7-14.0) years (Fig. 1b). Groups were too small to stratify by use of neoadjuvant treatment (only 10 patients with $<$ pT2N+after NAC). For the NCR cohort, median follow-up was 3.6 years with follow-up censored at 1 February 2017. For the NCR-BlaZIB cohort, median follow-up was 0.9 years with follow-up censored at 1 February 2020.

\section{Discussion}

Pathological downstaging to non-MIBC or pT0 at RC is a favorable prognostic factor. Nevertheless, we showed that LNM are present in 4.1 and $4.6 \%$ of patients with a complete downstaging [(y)pT0] or near-complete downstaging
Table 1 The prevalence of occult lymph node metastases in patients with cT2-4aN0M0 urothelial bladder cancer without evidence of residual muscle-invasive disease at radical cystectomy

\begin{tabular}{|c|c|c|c|}
\hline & pNO & $\mathrm{pN} 1-3$ & Total \\
\hline \multicolumn{4}{|l|}{ All patients } \\
\hline pT0 & 781 & $33(4.1 \%)$ & 814 \\
\hline $\mathrm{pTa} / \mathrm{is} / 1$ & 534 & $26(4.6 \%)$ & 560 \\
\hline$<\mathrm{pT} 2$ & 1315 & $59(4.3 \%)$ & 1374 \\
\hline \multicolumn{4}{|c|}{ cT2 $(N=4342)$} \\
\hline pT0 & 673 & $25(3.6 \%)$ & 698 \\
\hline $\mathrm{pTa} / \mathrm{is} / 1$ & 472 & $24(4.8 \%)$ & 496 \\
\hline$<\mathrm{pT} 2$ & 1145 & $49(4.1 \%)$ & 1194 \\
\hline \multicolumn{4}{|c|}{ cT3-4a $(N=1075)$} \\
\hline pT0 & 108 & $8(6.9 \%)$ & 116 \\
\hline pTa/is/1 & 62 & $2(3.1 \%)$ & 64 \\
\hline$<\mathrm{pT} 2$ & 170 & $10(5.6 \%)$ & 180 \\
\hline \multicolumn{4}{|c|}{ No NAC or NAR $(N=4798)$} \\
\hline pT0 & 585 & $20(3.3 \%)$ & 605 \\
\hline $\mathrm{pTa} / \mathrm{is} / 1$ & 486 & $24(4.7 \%)$ & 510 \\
\hline$<\mathrm{pT} 2$ & 1071 & $44(4.0 \%)$ & 1115 \\
\hline \multicolumn{4}{|c|}{$\mathrm{NAC}^{*}(N=513)$} \\
\hline урт0 & 171 & $8(4.5 \%)$ & 179 \\
\hline ypTa/is/1 & 42 & $2(4.6 \%)$ & 44 \\
\hline$<$ ypT2 & 213 & $10(4.5 \%)$ & 223 \\
\hline \multicolumn{4}{|c|}{$\mathrm{NAR}^{*}(N=108)$} \\
\hline урт0 & 25 & $5(16.7 \%)$ & 30 \\
\hline ypTa/is/1 & 7 & $0(0 \%)$ & 7 \\
\hline$<$ ypT2 & 32 & $5(13.5 \%)$ & 37 \\
\hline
\end{tabular}

$N A C$ neoadjuvant chemotherapy, NAR neoadjuvant radiotherapy

${ }^{*}$ Two patients received both NAC and NAR

[(y)pTa/is/1] of the primary tumor. This was regardless of the use of NAC. Moreover, these LNM were significantly associated with worse OS.

A systematic review by Bruins et al. indicated that any kind of PLND at RC is associated with beneficial OS versus no PLND [7]. Despite the low level of evidence, current guidelines recommend PLND as standard practice in combination with RC [1]. In patients who are not fit enough for $\mathrm{RC}$, refuse $\mathrm{RC}$ or prefer a bladder-sparing approach, treatment of the pelvic lymph nodes is usually not performed. In the 2019 EAU-ESMO Consensus Statements on the management of advanced and variant $\mathrm{BC}, 64 \%$ of the experts agreed that in $\mathrm{cN} 0$-disease, PLND in case of bladder preservation is not recommended [16]. In contrast, a similar percentage of experts agreed on radiation of the pelvic lymph nodes in case of trimodality treatment [16]. However, given the limited evidence available in current literature no definitive consensus could be reached for both statements.

In the randomized BC2001 trial [2], disease-free survival (DFS) was compared between patients with cT2-4aN0M0 


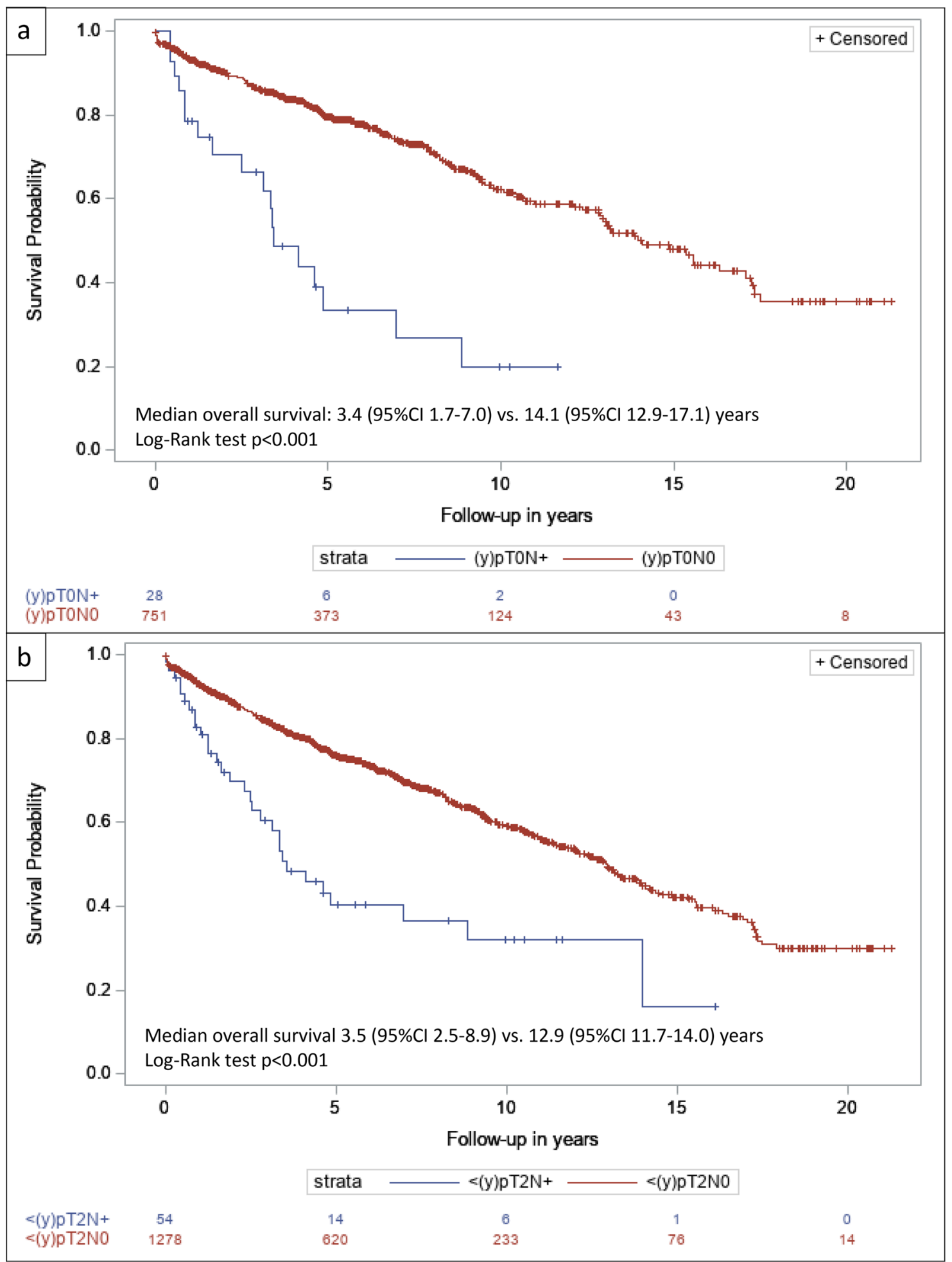

Fig. 1 a-b Overall survival of patients with and without occult lymph node metastases in cT2-4aN0M0 urothelial bladder cancer without evidence of residual bladder cancer [(y)pT0] at radical cystectomy (a) or residual muscle-invasive disease [(y)pT0/a/is/1] at radical cystectomy $(\mathbf{b})$

$\mathrm{BC}$ who underwent chemoradiotherapy (CMR) versus ERBT alone (radiation was confined to the bladder in both groups). The rate of lymph node relapses was not as high as might have been expected from surgical staging in $\mathrm{RC}$ cohorts, e.g., $4.9 \%(n=9)$ in the CMR group and $6.7 \%$ $(n=12)$ in the ERBT-only group [2]. In another randomized 
chemoradiotherapy trial (cT2-4N0M0), in which radiation of the whole pelvis was compared to radiation of the bladder alone, pelvic lymph node recurrences occurred in $15.8 \%$ (15/95) and $17.6 \%$ (16/91) of patients, respectively [17]. With a median follow-up of 5 years, OS and DFS did not significantly differ between groups. In the bladder only group the first draining lymph nodes might also have been irradiated since in general a $2 \mathrm{~cm}$ margin around the bladder is taken. Of note, differences in pelvic lymph node recurrences between the above mentioned trials might be due to the higher percentage of $\mathrm{T} 2$ patients at baseline in the BC2001 trail (83 vs. 46\%) [2, 17]. Several other, mostly retrospective studies on bladder-preserving strategies without EBRT or TMT following TUR (e.g., regimens of TURNAC-Re-TUR) did not report on the prevalence of LNM during follow-up and thereby do not address the potential role of PLND or treatment of the pelvic lymph nodes in such cases [18]. Since data on the survival effect of PLND in bladder-sparing approaches are not available, it would be interesting to compare morbidity and oncological outcomes for no treatment versus radiation versus minimal-invasive surgery for the pelvic lymph nodes in patients with MIBC undergoing bladder-preserving therapies with and without chemotherapy.

In the context of our study, it is important to note that the prevalence of LNM cannot simply be translated to the clinical scenario of selected patients with a presumed "pT0 status' after a TUR with or without NAC and Re-TUR. Given significant discrepancies in residual tumor and LNM rates between a presumed ' $(y)$ pT0 status' and a confirmed pT0disease in RC specimens $[9,10]$, our results might indicate an underestimation of the prevalence of LNM in patients who are treated with TUR and/or NAC only. For example, in our RC cohort, occult LNM were present in $13 \%$ of patients with pT2-disease. Moreover, in our study PLND templates were not available, which might further underestimate the true prevalence of LNM. An earlier published NCR study indicated evidence of PLND template extension in more recent study years, as was shown by a higher number of LNM in patients with comparable clinical disease characteristics over time [19]. In line with these findings, pelvic and sentinel lymph node mapping studies in BC confirm that a limited versus an extended PLND does not capture all draining lymph nodes and thus might lead to a false negative 'pN0 status' [20, 21]. It is, therefore, likely that the true prevalence of LNM in patients with a presumed 'pT0 status' before RC is higher than the 5\% which was found in both the study of Nassiri et al. [11] and our study. This assumption might favor the harm to benefit ratio to perform a diagnostic PLND. Although the survival benefit of PLND in this particular group of patients is unknown, the outcome may guide adjuvant treatment. The CheckMate 274 study showed improved DFS in patients with lymph node-positive disease after NAC plus RC and PLND treated with adjuvant nivolumab [22].

It can be questioned if there are viable alternatives to a PLND or tools to select patients for whom a PLND is appropriate. The vast majority of patients in our database was staged with a contrast enhanced $\mathrm{CT}$ of the abdomen and a CT or conventional X-ray of the chest. Mertens et al. recently showed that by use of a FDG-PET-CT, $21 \%$ of patients were upstaged to non-localized disease [23]. Half of this group was upstaged due to regional nodal metastases. The other half had supraregional nodal or distant metastases. Clinical management changed in $13.5 \%$ of patients as a result of upstaging defined by FDG-PET-CT [23]. More sensitive imaging modalities, like FDG-PET-CT, might better select patients for PLND treated within a bladder-sparing treatment protocol. Still, according to a systematic review and meta-analysis by $\mathrm{Ha}$ et al. the pooled sensitivity for the detection of LNM by FDG-PET-CT was only 57\% [24]. One could also argue if a sentinel node ( $\mathrm{SN}$ ) procedure could have a role in whether or not to proceed with PLND, thereby minimizing surgical risks. In $\mathrm{BC}$, the reported $\mathrm{SN}$ detection rates range from 81 to $92 \%$. However, in initial validation studies false negative rates up to $19 \%$ were reported [25]. In a recent single center study, Zarifmahmoudi et al. reported a SN detection rate of $85 \%$ and a false negative rate as high as $42 \%$ [26]. However, another MIBC study concluded that $\mathrm{SN}$ detection played no role in staging of nodal disease since the vast majority of LNM were detected in the non-sentinel lymph nodes [27]. The high number of false negatives would, therefore, lead to understaging if one does not proceed with PLND if the outcome of the SN is negative. Altogether, prospective research in promising imaging modalities and minimally invasive diagnostics is needed to further clarify the role of PLND in bladder-sparing treatment protocols in which PLND is not standard of care.

The presence of circulating tumor cells (CTCs) in patients with muscle-invasive bladder cancer is another promising area of research. A recently presented abstract from the CirGuidance study, evaluating the role of CTCs in relation to response to NAC, showed promising results: CTC-positive patients had better overall survival when they received NAC [29]. However, the full content of this study is not yet published. It would be of interest to know whether the presence of CTCs is also predictive for occult LNM in patient with and without NAC.

Our study is subject to several limitations. In the earlier cohort, data were retrospectively collected in contrast with the more recent prospective NCR-BlaZIB cohort. Despite the high number of RCs, the group of patients with (near) complete downstaging and the presence of LNM remained low. Also, information on neoadjuvant treatment was limited. In case of NAC, exact regimens and the number of cycles were unknown. This was the same for radiation 
schemes in the NAR-group. Recent changes in preoperative diagnostic modalities, e.g., the use of more sensitive imaging like FDG-PET scans might result in a Will Rogers phenomenon [28]. Unfortunately, our databases had no information available regarding the use of FDG-PET scans versus conventional CT scans. Therefore, we could not assess the primary study outcome stratified by different preoperative imaging modalities. However, since the prevalence of occult LNM was similar between cohorts (NCR cohort: $4.2 \%$, NCR-BlaZIB cohort: $4.6 \%$ ) we expect the impact of such stage migration to be minimal. No information was available on the extent of the PLND templates. Since a limited PLND was often performed in the past, it is likely that we underestimated the true prevalence of occult nodal metastasis in this study. This may, however, further strengthen the potential role of PLND in selected patients who do not undergo RC. In addition, this emphasizes the need for future research to evaluate, for example, the extent of the PLND template, lymph node density in positive cases and extracapsular extension in lymph nodes and their effects on prognosis and adjuvant treatments. Also, it will be important to identify risk factors predicting the presence of occult LNM after downstaging of the primary tumor (e.g., lymphovascular invasion, perineural spread, Ki-67 index on TURBT), as this might influence treatment decision-making as well. Despite these limitations, this is the second large nationwide database study to report on the prevalence of LNM in the patients with bladder cancer that were downstaged to (y)pT0 or (y)pTa/is/1 disease in the $\mathrm{RC}$ specimen.

\section{Conclusion}

After RC and PLND for cT2-4aN0M0 urothelial BC, occult LNM occur in $4.3 \%$ of patients with a (near)-complete downstaging of the primary tumor. This was regardless of NAC or initial clinical T-stage. Patients with occult LNM showed considerable worse survival. The risk of occult LNM should be considered and discussed with patients opting for bladder-sparing treatment. Future research, therefore, should address the diagnostic and therapeutic value of PLND in patients with MIBC undergoing bladder-sparing treatment protocols (e.g., TUR-only \pm NAC, EBRT or TMT). Consequently, the outcome of PLND may have implications for radiation field extension, adjuvant treatment with chemotherapy or immune checkpoint inhibitors.

Supplementary Information The online version contains supplementary material available at https://doi.org/10.1007/s00345-021-03839-7.

Acknowledgements The authors thank the registration team of the Netherlands Comprehensive Cancer Organisation (IKNL) for the collection of data for the Netherlands Cancer Registry as well as IKNL staff for their help, the Dutch Uro-Oncology Study group (DUOS) and the Dutch Cancer Society (KWF Kankerbestrijding) for the financial support and the Dutch Pathology Registry (PALGA) for their support in the data acquisition (in particular, Hester $\mathrm{H}$. van Boven, $\mathrm{MD}, \mathrm{PhD}$, Pathologist at the Netherlands Cancer Institute-Antoni van Leeuwenhoek Hospital and Rinus Voorham, PALGA data manager).

The members of the BlaZIB study group are: Katja K.H. Aben (PI), $\mathrm{PhD}$ (Netherlands Comprehensive Cancer Organisation, Radboud University Medical Center). Lambertus A.L.M. Kiemeney (PI), PhD, Prof (Radboud University Medical Center). J. Alfred Witjes (PI), MD, PhD, Prof (Radboud University Medical Center). Lisa M.C. van Hoogstraten, MSc (Netherlands Comprehensive Cancer Organisation). Theodora M. Ripping, $\mathrm{PhD}$ (Netherlands Comprehensive Cancer Organisation). Joost Boormans, MD, PhD (Erasmus Medical Center). Catharina A. Goossens-Laan, MD, PhD (Alrijne hospital). Sipke Helder (Patient association 'Leven met blaas- of nierkanker). Maarten C.C.M. Hulshof, $\mathrm{MD}, \mathrm{PhD}$ (Amsterdam University Medical Centers, University of Amsterdam). Geert J.L.H. van Leenders, MD, PhD (Erasmus Medical Center). Anna M. Leliveld, MD, PhD (University Medical Center Groningen). Richard P. Meijer, MD, PhD (University Medical Center Utrecht). Sasja F. Mulder, MD, PhD (Radboud University Medical Center). Ronald I. Nooter (Franciscus Gasthuis \& Vlietland hospital). Juus L. Noteboom, MD, PhD (University Medical Center Utrecht). Jorg R. Oddens, MD, PhD (Amsterdam University Medical Centers, University of Amsterdam). Theo M. de Reijke, MD, PhD (Amsterdam University Medical Centers, University of Amsterdam). Tineke J. Smilde, MD, PhD (Jeroen Bosch ziekenhuis). Guus W.J. Venderbosch (Patient association 'Leven met blaas- of nierkanker'). Antoine G. van der Heijden, MD, PhD (Radboud University Medical Center). Michiel S. van der Heijden, MD, PhD (Netherlands Cancer Institute). Reindert J.A. van Moorselaar, MD, PhD, Prof (Amsterdam University Medical Centers, Vrije Universiteit Amsterdam). Bas W.G. van Rhijn, MD, $\mathrm{PhD}$, FEBU (Netherlands Cancer Institute - Antoni van Leeuwenhoek Hospital). Joep G.H. van Roermund, MD, PhD (Maastricht University Medical Center). Bart P. Wijsman, MD, PhD (Elisabeth-TweeSteden Ziekenhuis)

Author contributions TJN Hermans, BWG van Rhijn, KKH Aben, and LMC van Hoogstraten contributed to the study conception and design. Material preparation and data collection were performed by LMC van Hoogstraten, LALM Kiemeney LALM, JA Witjes, CS Voskuilen, M Deelen, LS Mertens, TM Ripping, BWG van Rhijn, KKH Aben, and TJN Hermans. Analysis was performed by TJN Hermans and LMC van Hoogstraten. The first draft of the manuscript was written by TJN Hermans, LMC van Hoogstraten, and EJ van Gennep and all authors commented on previous versions of the manuscript. All authors read and approved the final manuscript.

Funding The BlaZIB study is funded by the Dutch Cancer Society (KWF, IKNL 2015-7914). The funding agency had no further role in this study.

Availability of data and material All data used for this study can be requested from the NCR. All data requests are reviewed by the supervisory committee of the NCR for compliance with the NCR objectives and (inter)national (privacy) regulation and legislation.

Code availability Code used for this study can be made available post publication by the authors upon request.

\section{Declarations}

Conflict of interest The authors have no competing interests to declare. 
Ethical approval According to the Central Committee on Research involving Human Subjects (CCMO), this type of study does not require approval from an ethics committee in the Netherlands. The requirement for informed consent was waived due of the retrospective design of the study. This study was approved by the Netherlands Cancer Registry's Supervisory Committee.

Open Access This article is licensed under a Creative Commons Attribution 4.0 International License, which permits use, sharing, adaptation, distribution and reproduction in any medium or format, as long as you give appropriate credit to the original author(s) and the source, provide a link to the Creative Commons licence, and indicate if changes were made. The images or other third party material in this article are included in the article's Creative Commons licence, unless indicated otherwise in a credit line to the material. If material is not included in the article's Creative Commons licence and your intended use is not permitted by statutory regulation or exceeds the permitted use, you will need to obtain permission directly from the copyright holder. To view a copy of this licence, visit http://creativecommons.org/licenses/by/4.0/.

\section{References}

1. Witjes JA, Bruins HM, Cathomas R, Compérat EM, Cowan NC, Gakis G et al (2021) European Association of Urology guidelines on muscle-invasive and metastatic bladder cancer: summary of the 2020 guidelines. Eur Urol 79(1):82-104

2. James ND, Hussain SA, Hall E, Jenkins P, Tremlett J, Rawlings C et al (2012) Radiotherapy with or without chemotherapy in muscle-invasive bladder cancer. N Engl J Med 366(16):1477-1488

3. Solsona E, Iborra I, Collado A, Rubio-Briones J, Casanova J, Calatrava A (2010) Feasibility of radical transurethral resection as monotherapy for selected patients with muscle invasive bladder cancer. J Urol 184(2):475-480

4. Mazza P, Moran GW, Li G, Robins DJ, Matulay JT, Herr HW et al (2018) Conservative management following complete clinical response to neoadjuvant chemotherapy of muscle invasive bladder cancer: contemporary outcomes of a multi-institutional cohort study. J Urol 200(5):1005-1013

5. Audenet F, Waingankar N, Ferket BS, Niglio SA, Marqueen KE, Sfakianos JP et al (2018) Effectiveness of transurethral resection plus systemic chemotherapy as definitive treatment for muscle invasive bladder cancer in population level data. $\mathbf{J}$ Urol 200(5):996-1004

6. Hermans TJ, Fransen van de Putte EE, Horenblas S, van Rhijn BW, Verhoeven RH (2016) Extended pelvic lymph node dissection at radical cystectomy for bladder cancer improves survival: results of a nationwide population-based study. Int J Urol 23(12):1043-1044

7. Bruins HM, Veskimae E, Hernandez V, Imamura M, Neuberger MM, Dahm P et al (2014) The impact of the extent of lymphadenectomy on oncologic outcomes in patients undergoing radical cystectomy for bladder cancer: a systematic review. Eur Urol 66(6):1065-1077

8. Hermans TJN, Voskuilen CS, Deelen M, Mertens LS, Horenblas S, Meijer RP et al (2019) Superior efficacy of neoadjuvant chemotherapy and radical cystectomy in cT3-4aN0M0 compared to cT2N0M0 bladder cancer. Int J Cancer 144(6):1453-1459

9. Kukreja JB, Porten S, Golla V, Ho PL, Noguera-Gonzalez G, Navai N et al (2018) Absence of tumor on repeat transurethral resection of bladder tumor does not predict final pathologic $\mathrm{T} 0$ stage in bladder cancer treated with radical cystectomy. Eur Urol Focus 4(5):720-724

10. deVere White RW, Lara PN Jr, Goldman B, Tangen CM, Smith DC, Wood DP Jr et al (2009) A sequential treatment approach to myoinvasive urothelial cancer: a phase II Southwest Oncology Group trial (S0219). J Urol 181(6):2476-2480

11. Nassiri N, Ghodoussipour S, Maas M, Nazemi A, Asanad K, Pearce S et al (2020) occult nodal metastases in patients downstaged to nonmuscle invasive disease following neoadjuvant chemotherapy. Urology 142:155-160

12. Ripping TM, Kiemeney LA, van Hoogstraten LMC, Witjes JA, Aben KKH (2020) Insight into bladder cancer care: study protocol of a large nationwide prospective cohort study (BlaZIB). BMC Cancer 20(1):455

13. Casparie M, Tiebosch AT, Burger G, Blauwgeers H, van de Pol A, van Krieken JH et al (2007) Pathology databanking and biobanking in The Netherlands, a central role for PALGA, the nationwide histopathology and cytopathology data network and archive. Cell Oncol 29(1):19-24

14. Fritz A, Jack A, Percy C, Sobin L, Shanmugarathan S, Whelan $\mathrm{S}$. International classification of diseases for oncology: ICD-O: World Health Organization; 2000.

15 Brierley JD, Gospodarowicz MK, Wittekind C (2017) TNM classification of malignant tumours. Wiley

16. Horwich A, Babjuk M, Bellmunt J, Bruins HM, De Reijke TM, De Santis M et al (2019) EAU-ESMO consensus statements on the management of advanced and variant bladder cancer-an international collaborative multi-stakeholder effort: under the auspices of the EAU and ESMO Guidelines Committees $\dagger$. Ann Oncol 30(11):1697-1727

17. Tunio MA, Hashmi A, Qayyum A, Mohsin R, Zaeem A (2012) Whole-pelvis or bladder-only chemoradiation for lymph nodenegative invasive bladder cancer: single-institution experience. Int J Radiat Oncol Biol Phys 82(3):e457-e462

18. Moran GW, Li G, Robins DJ, Matulay JT, McKiernan JM, Anderson CB (2017) Systematic review and meta-analysis on the efficacy of chemotherapy with transurethral resection of bladder tumors as definitive therapy for muscle invasive bladder cancer. Bladder Cancer 3(4):245-258

19. Hermans TJ, Fransen van de Putte EE, Fossion LM, Werkhoven EV, Verhoeven RH, van Rhijn BW et al (2016) Variations in pelvic lymph node dissection in invasive bladder cancer: a Dutch nationwide population-based study during centralization of care. Urol Oncol 34(12):532

20. Dorin RP, Daneshmand S, Eisenberg MS, Chandrasoma S, Cai J, Miranda G et al (2011) Lymph node dissection technique is more important than lymph node count in identifying nodal metastases in radical cystectomy patients: a comparative mapping study. Eur Urol 60(5):946-952

21. Roth B, Wissmeyer MP, Zehnder P, Birkhäuser FD, Thalmann GN, Krause TM et al (2010) A new multimodality technique accurately maps the primary lymphatic landing sites of the bladder. Eur Urol 57(2):205-211

22. UroToday.com. ASCO GU 2021: First Results from the Phase 3 CheckMate 274 Trial of Adjuvant Nivolumab vs Placebo in Patients Who Underwent Radical Surgery for High-Risk Muscle-Invasive Urothelial Carcinoma.

23. Mertens LS, Fioole-Bruining A, Vegt E, Vogel WV, van Rhijn BW, Horenblas S (2013) Impact of (18) F-fluorodeoxyglucose (FDG)-positron-emission tomography/computed tomography (PET/CT) on management of patients with carcinoma invading bladder muscle. BJU Int 112(6):729-734

24. Ha HK, Koo PJ, Kim SJ (2018) Diagnostic accuracy of F-18 FDG PET/CT for preoperative lymph node staging in newly diagnosed bladder cancer patients: a systematic review and meta-analysis. Oncology 95(1):31-38 
25. Liedberg F, Chebil G, Davidsson T, Gudjonsson S, Månsson W (2006) Intraoperative sentinel node detection improves nodal staging in invasive bladder cancer. J Urol 175(1):84-88

26. Zarifmahmoudi L, Ghorbani H, Sadeghi R, Sadri K, Tavakkoli M, Keshvari M et al (2020) Sentinel lymph node biopsy in muscle-invasive bladder cancer: single-center experience. Ann Nucl Med 34(10):718-724

27. Alvaeus J, Rosenblatt R, Johansson M, Alamdari F, Jakubczyk T, Holmström B et al (2020) Fewer tumour draining sentinel nodes in patients with progressing muscle invasive bladder cancer, after neoadjuvant chemotherapy and radical cystectomy. World J Urol 38(9):2207-2213
28. Sormani MP (2009) The Will Rogers phenomenon: the effect of different diagnostic criteria. J Neurol Sci 287(Suppl 1):S46-S49

29. UroToday.com. EAU 2021: Circulating Tumor Cell-Driven Use of Neoadjuvant Chemotherapy in Patients With Muscle-Invasive Bladder Cancer: Final Results of the CirGuidance Study.

Publisher's Note Springer Nature remains neutral with regard to jurisdictional claims in published maps and institutional affiliations.

\section{Authors and Affiliations}

\section{M. C. van Hoogstraten ${ }^{1,4}\left(\right.$ ) E. J. van Gennep ${ }^{2}$. L. A. L. M. Kiemeney ${ }^{3,4}$. J. A. Witjes ${ }^{3}$. C. S. Voskuilen ${ }^{5}$ M. Deelen ${ }^{6}$ L. S. Mertens ${ }^{5}$. R. P. Meijer ${ }^{7}$. J. L. Boormans ${ }^{8}$. D. G. J. Robbrecht ${ }^{9} \cdot$ L. V. Beerepoot ${ }^{10} \cdot$ R. H. A. Verhoeven ${ }^{1,11}$. T. M. Ripping ${ }^{1}$ B BlaZIB Study Group • B. W. G. van Rhijn ${ }^{12} \cdot$ K. K. H. Aben ${ }^{1,4}$ •T. J. N. Hermans ${ }^{13}$}

1 Department of Research and Development, Netherlands Comprehensive Cancer Organisation, Utrecht, The Netherlands

2 Department of Urology, Leiden University Medical Center, Leiden, The Netherlands

3 Department of Urology, Radboud University Medical Center, Nijmegen, The Netherlands

4 Radboud University Medical Center, Radboud Institute for Health Sciences, Nijmegen, The Netherlands

5 Department of Urology, Netherlands Cancer InstituteAntoni Van Leeuwenhoek Hospital, Amsterdam, The Netherlands

6 Department of Urology, Maastricht University Medical Center+, Maastricht, The Netherlands

7 Department of Oncological Urology, University Medical Center Utrecht, Utrecht, The Netherlands
8 Department of Urology, Erasmus University Medical Center Rotterdam, Rotterdam, The Netherlands

9 Department of Medical Oncology, Erasmus Medical Center Cancer Institute, Rotterdam, The Netherlands

10 Department of Medical Oncology, Elisabeth-TweeSteden Hospital, Tilburg, The Netherlands

11 Department of Medical Oncology, Cancer Center Amsterdam, Amsterdam University Medical Centers, University of Amsterdam, Meibergdreef 9, 1105, AZ, Amsterdam, The Netherlands

12 Department of Surgical Oncology (Urology), Netherlands CancerInstitute-Antoni Van Leeuwenhoek, Amsterdam, The Netherlands

13 Department of Urology, Catharina Hospital Eindhoven, Eindhoven, The Netherlands 